\title{
Tabletop mid-infrared nulling testbed for the Keck interferometer and the Terrestrial Planet Finder
}

Christopher D. Koresko, Bertrand P. Mennesson, Samuel L. Crawford, Michelle J. Creech-Eakman, J. Kent Wallace, et al.

Christopher D. Koresko, Bertrand P. Mennesson, Samuel L. Crawford, Michelle J. Creech-Eakman, J. Kent Wallace, Eugene Serabyn, "Tabletop mid-infrared nulling testbed for the Keck interferometer and the Terrestrial Planet Finder," Proc. SPIE 4838, Interferometry for Optical Astronomy II, (21 February 2003); doi: 10.1117/12.458017

Event: Astronomical Telescopes and Instrumentation, 2002, Waikoloa, Hawai'i, United States 


\title{
A Tabletop Mid-Infrared Nulling Testbed for the Keck Interferometer and the Terrestrial Planet Finder
}

\author{
Chris Koresko ${ }^{a}$, Bertrand Mennesson ${ }^{b}$, Samuel Crawford ${ }^{b}$, \\ Michelle Creech-Eakman ${ }^{b}$, Kent Wallace ${ }^{b}$, Eugene Serabyn ${ }^{b}$ \\ ${ }^{a}$ Interferometry Science Center, Caltech, Pasadena, CA, USA \\ ${ }^{b}$ Jet Propulsion Laboratory, Caltech, Pasadena, CA, USA
}

\begin{abstract}
A tabletop rotational-shearing interferometer experiment has been constructed and operated at JPL to serve as a testbed for the mid-infrared $(\sim 10 \mu \mathrm{m})$ nulling beam combiners on the Keck Interferometer and the Terrestrial Planet Finder. The testbed is a pupil-plane combiner in which destructive combination of the incoming wavefronts is achieved using a rooftop mirror system in which the polarization vector is flipped along the vertical axis on one arm and the horizontal axis on the other. The optical pathlength along one arm is adjustable using a linear stage driven by picomotor and piezoelectric actuators. The combined light is focussed onto a single-pixel $\mathrm{LN}_{2}$-cooled $\mathrm{HgCdTe}$ detector. In order to provide adequate sensitivity in the presence of the very bright thermal emission from the room-temperature optics, the light source is modulated and the output is demodulated using a lock-in amplifier. The optical pathlength difference (OPD) is stabilized under computer control by slowly dithering the actuated arm and balancing the leakage signal on either side of the null. The system has produced a stabilized null depth of $<10^{-4}$ using a diode laser source emitting at a wavelength of $9.2 \mu \mathrm{m}$, and transient nulls of $10^{-2}$ with a broadband thermal IR source in a $6.4 \%$ optical bandpass.
\end{abstract}

Keywords: Interferometry, Nuller, Astronomy, Infrared, Laboratory

\section{INTRODUCTION}

The introduction of a 180-degree relative rotational shear, or "field flip", into the inputs of a pupil-plane beam combiner has been one of the favored approaches for producing deep achromatic nulls for the mid-infrared (7-17 $\mu \mathrm{m})$ version of the Terrestrial Planet Finder (TPF) mission ${ }^{1}$. A deep null centered on a bright star would permit detection and spectroscopy of the $\sim 10^{6}$ times fainter light coming from an Earthlike planet at an angular distance of only $\sim 0^{\prime \prime} .1$ from the star.

In order to explore the use of a "field-flip" nulling beam combiner ("nuller") at mid-infrared wavelengths, we have constructed and operated a tabletop demonstrator at JPL. The basic design of the instrument is very similar to that which has previously been used successfully at visible wavelengths ${ }^{2}$. The fundamental idea is straightforward. In brief, the two end mirrors in a typical Michelson-style interferometer are replaced with rooftop mirrors which are oriented such that the "spine" of one rooftop is perpendicular to that of the other. Reflection by each rooftop reverses the sign of the vector component of the electric field which lies perpendicular to the spine. As a result, the beam combination occurs after the field in one arm has been flipped in the horizontal axis while the field in the other arm has been flipped in the vertical axis, so that for zero optical pathlength difference (OPD) the overall electric field vectors are opposed at the point of combination. This should in principle produce an achromatic null fringe. An additional flat mirror is inserted into each arm in order to balance the total number of s-plane and p-plane reflections. A schematic of the optical layout is shown in Figure 1.

This instrument has reached a null depth, defined as the ratio of the output intensity at the destructive fringe to that at the constructive fringe, of $<10^{-4}$ using a diode laser source at a wavelength of $9.2 \mu \mathrm{m}$, and sustained them using a simple pathlength-dithering servo system. Transient nulls as deep as $10^{-2}$ have been produced with a broadband thermal infrared source. This paper describes the instrument and offers some general conclusions about the usefulness of this rooftop field-flip approach for nulling in the thermal infrared. Because the instrument described here is closely related to the visible-light rooftop nuller, the emphasis will be on those aspects which are unique to operation at the longer wavelengths. 


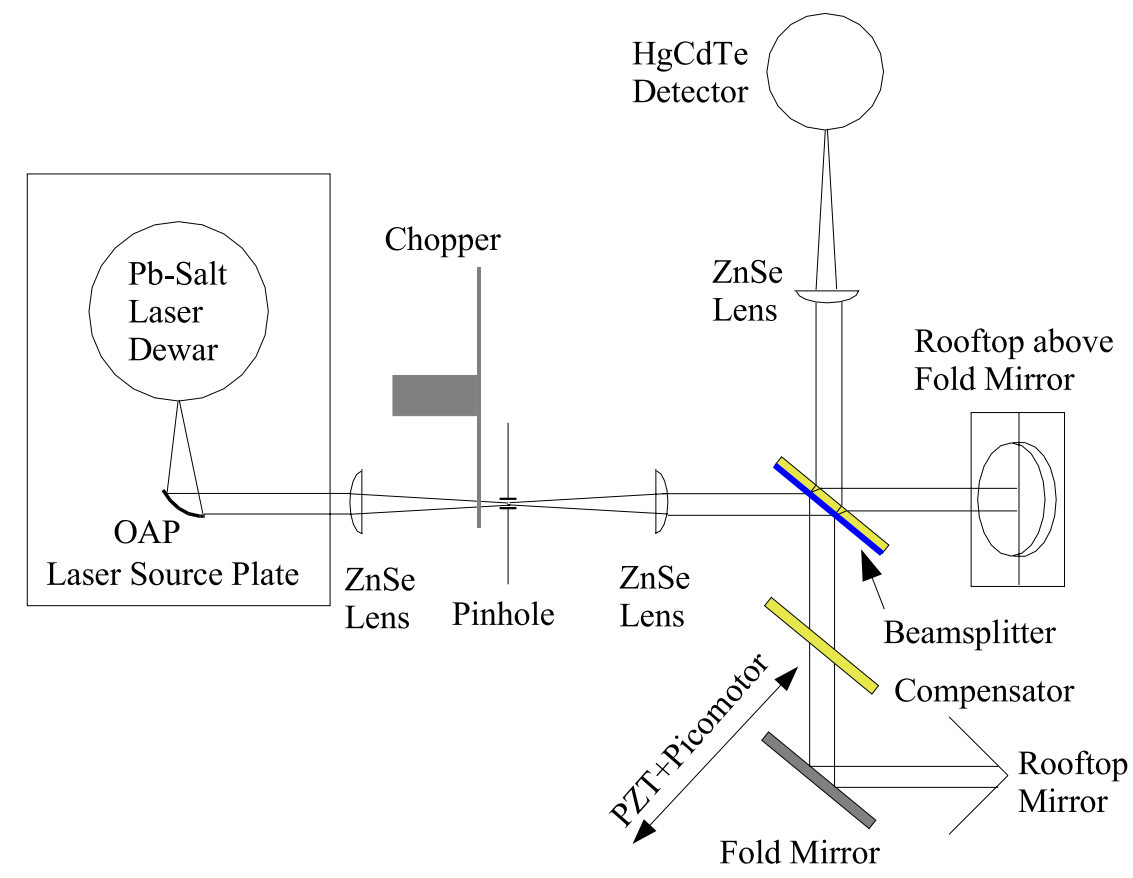

Figure 1. Tabletop nuller optical system configured with the diode laser as a light source. The light is collimated by an off-axis paraboloid, modulated by a rotating chopper wheel near the focal plane, and focussed onto a pinhole which acts as a spatial filter. The filtered light is then recollimated and split, with approximately half of the photons sent to each of the two arms. Each arm reverses one vector component of the polarization, so that when the beams are recombined at the beamsplitter, the interference is achromatically destructive when the OPD is zero. The pathlength in one arm can be adjusted by moving its fold mirror in the direction shown, under control of either a picomotor or a PZT actuator.

\section{OPTICAL SYSTEM}

The optical design of the apparatus described in this paper is conceptually a straightforward adaptation of the existing visible-light rooftop nuller design ${ }^{2}$. Changes relative to the previous design were driven mainly by the requirement to produce, transmit, and detect light at thermal infrared rather than visible wavelengths. The optical changes involved the use of a pinhole rather than a single-mode fiber for spatially filtering the input light source (since no adequate single-mode fibers usable at $10 \mu \mathrm{m}$ were available), bare gold reflecting surfaces (for high throughput and minimization of polarization effects), and ZnSe transmitting optics (lenses, the beamsplitter substrate, and its compensator).

The basic layout of the optical system is shown in Figure 1. As shown, the system is set up to use a mid-infrared diode laser as a light source, and to modulate it using a chopper wheel. Experiments were done with several additional configurations. These differ from the one shown only in their input and output. For example, for certain tests a wire-grid linear polarizer was inserted in the collimated space between the source and the beamsplitter. For broadband testing, the laser source was sometimes replaced by a thermal emitter and collimating lens. A second spatial filter (pinhole and focusing lens) just ahead of the detector was sometimes installed. Various objects were placed in the arms to attenuate the brighter beam and thereby match the fluxes in the two arms. Throughout these tests, however, the heart of the system, consisting of the beamsplitter, compensator, fold mirrors, and rooftops, remained intact. A photograph of the system in a slightly different configuration is shown in Figure 2 


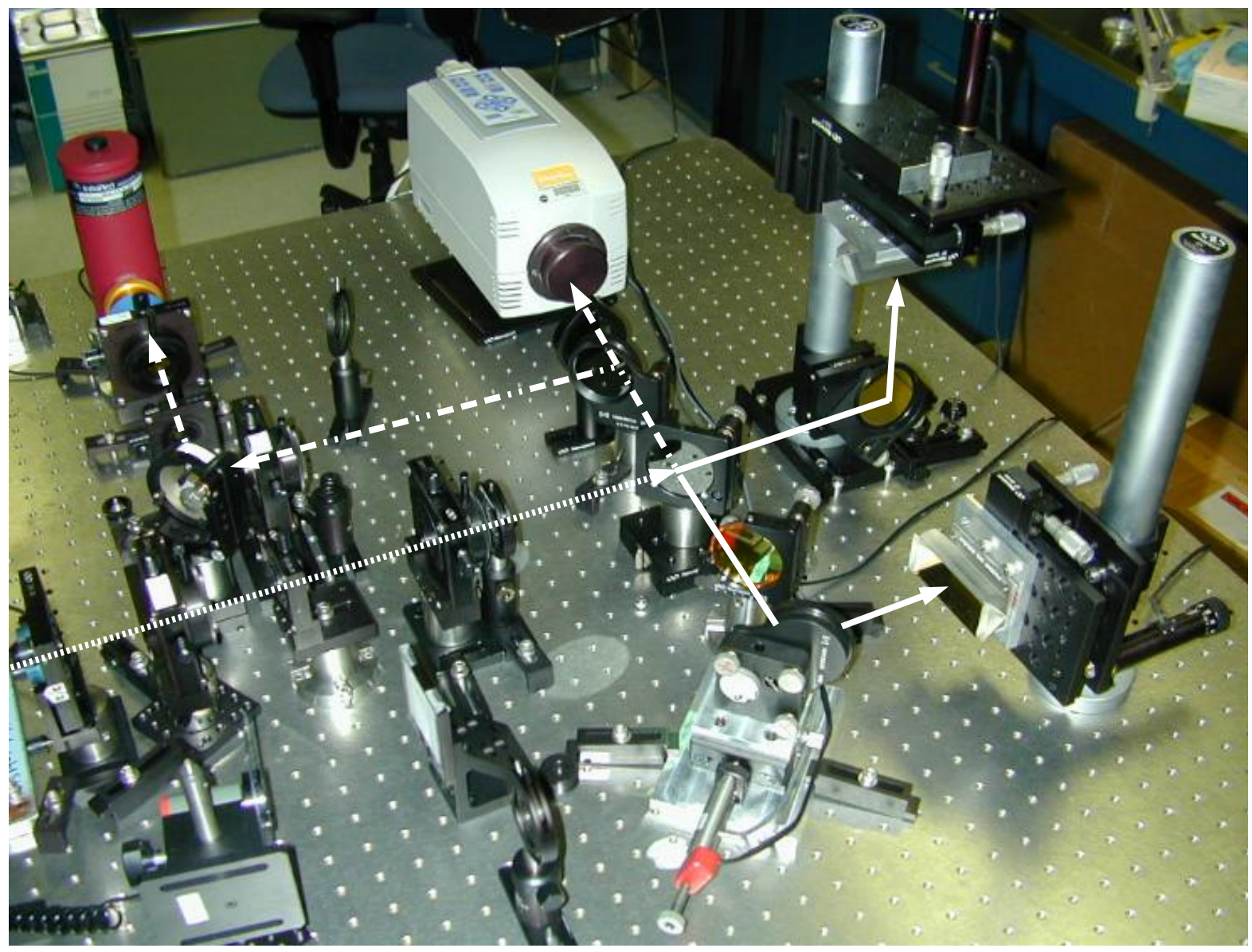

Figure 2. Photograph of the tabletop infrared nuller. The actual nulling interferometer is toward the lower right, with collimated light entering the system from the lower left (the light source is not shown). The linear polarizer is at the left edge of the image, followed by the spatial filter (focussing lens, pinhole, and recollimating lens). The beamsplitter, whose active surface faces the photographer, sends approximately half the light into the horizontal arm in the lower right of the picture. The picomotor+PZT actuator for the fold mirror in the horizontal arm is in the bottom center. The input light which passes thorugh the beamsplitter into the vertical arm is reflected upward to a down-looking rooftop mirror. The light is recombined at the beamsplitter. At the time this picture was taken, the combined light was sent to a second beamsplitter which divided it between the $\mathrm{HgCdTe}$ photodiode detector described in the text, and a Stirling-cooled quantum well infrared array camera (the large white box at the top center) which was used for alignment. The white lines trace the light path, with the input light indicated with a dotted lines, the split light with solid lines, and the recombined light with dashes. Arrowheads point to the two rooftop mirrors and the two detectors. 


\section{LIGHT SOURCES AND DETECTOR}

Both a broadband unpolarized thermal emitter and a narrowband polarized laser source were used for the experiment. Although the thermal source more closely approximates the light from an astrophysical target such as a star, dust disk, or planet, several factors make achieving a deep null much more challenging with it. Chromatic dispersion due to unbalanced pathlengths through the various dispersive components in the system (including coatings and the substrates of transmissive optics) is expected to be a significant contributor to the leakage through the null. Vertically and horizontally polarized components of light suffer different phase delays as they pass through dielectric stacks which are not perpendicular to the propagation direction. Both of these effects, which cause the null fringe to be blurred because they make the fringe position dependent on wavelength and polarization state, are greatly alleviated when the light is narrowband and vertically polarized. In addition, it is not trivial to reach sufficient sensitivity to measure and track a deep null on a thermal source. This is because the interferometer has a very narrow field of view (limiting the product $A \Omega$ of the emitting area and the solid angle into which photons emitted by it will be captured by the optical system) while the temperature of the emitting material limits the intensity of its emission.

The thermal source chosen for our experiment is a commercial hot-filament light source made by Cal Sensors, in which the usual glass bulb has been replaced by a metal can with a hermetically-sealed window. The window material is chosen to be transparent in the thermal infrared. We experimented with several window materials (ZnSe, antireflection-coated $\mathrm{Ge}$, and $\mathrm{CaF}_{2}$ ) and found that the detected signal was weakest for the $\mathrm{CaF}_{2}$ unit (the window cuts off the long-wavelength end of the bandpass of the detector) and about equally strong for the other two. We chose to use the ZnSe unit because the window is transparent at visual wavelengths, making it convenient to see when the source was turned on and to visually estimate the temperature of the filament from its color. The nominal operating temperature of the filament is $1170 \mathrm{~K}$. We found that it was possible to safely operate the unit with significantly more than its rated electrical current. However, because the optical passband is in the long-wavelength regime of the source's blackbody curve, its intensity is only linearly dependent on the filament temperature and overdriving the source provided only a modest boost in the detected signal.

The narrowband source used was a commercial Boston Electronics lead-salt diode laser system using lasers made by Laser Components. This $\mathrm{LN}_{2}$-cooled system produced a collimated beam at a wavelength of $9.2 \mu \mathrm{m}$, with a power of approximately $300 \mu \mathrm{W}$. Its output beam was partially vertically polarized. The beam was collimated by an off-axis paraboloidal mirror which was included with the unit.

The experiment calls for a spatially-filtered, collimated source. This was produced by using a positive lens to make an image of the source on a pinhole, and a second positive lens one focal length further downstream to collimate the light. In practice, there is a tradeoff between the quality of the spatial filtering and the throughput, and several pinhole sizes were tried.

The detector used was a commercial IR Associates $\mathrm{LN}_{2}$-cooled $\mathrm{HgCdTe}$ photodiode enclosed in a small dewar. The dewar was modified by adding a filter behind the input window to limit the sensitive optical bandpass to $\sim 30 \%$ centered at $\sim 10 \mu \mathrm{m}$, and an aperture was added to restrict the input beam to a focal ratio of $\mathrm{f} / 5$. Noise intrinsic to the detector, rather than the shot-noise statistics of the thermal infrared background, limited its sensitivity. The advantage of this detector is that it is small (the dewar body is about the size of a soda can), inexpensive, and convenient to use (it reaches its cryogenic operating temperature within about 15 minutes of being filled). The detector is connected to a commercial preamplifier. Its overall sensitivity is not optimal (i.e., it is not background-limited, even with the rather broad optical bandpass). In order to improve sensitivity, we have since obtained a new LHe-cooled single-pixel detector, but this was not operational at the time of the experiments described here.

The mid-infrared wavelength regime differs from the visible in that everything in a room-temperature lab, including the optical system itself emits strongly, and the photon flux is generally huge. As a result, the noise in a measurement tends to be large and independent of the signal even with an ideal detector. It is therefore necessary to modulate the light source and synchronously demodulate the detected signal. This restricts the range of frequency and phase to which the system is sensitive, and in typical conditions can greatly improve the overall sensitivity. 
Two source modulation schemes were tried. The first was to insert a mechanical chopper wheel close to the focus at the position of the input pinhole. We modulated the light from the emitter at a frequency close to 800 Hz. The chopper controller provided TTL synchronization pulses with the same frequency as the modulation and at a constant phase relative to it. These pulses were fed to the reference input of a commercial lock-in amplifier (Stanford Research SR830).

The second scheme for modulating the infrared emission was used only for the laser source. The laser controller had an analog input which controlled the current to the laser. The lock-in amplifier was set to produce its own sine-wave reference signal, and this was connected to the laser controller's current control input. The modulation frequency could be varied over a wide range without much effect on the experiment.

\section{DATA SYSTEM AND SERVO}

The preamplified detector signal was connected to the signal input of the lock-in. The lock-in amplifier used for this experiment was a digital unit equipped with a standard RS-232 serial interface through which many parameters could be controlled and the output voltage read. In addition, the unit has a set of analog outputs which can also be controlled via the serial interface. One of these analog outputs was connected to the input of the PZT actuator controller.

The lock-in amplifier was connected through this serial interface to a Linux-based personal computer. The computer's parallel port, which produces a set of TTL voltages controllable by writing to an I/O port address, was connected to the control input of the drive electronics box which controlled the picomotor actuator. The personal computer could therefore command motions of the OPD stage, set up the amplifier parameters, and read the amplitude of the resulting demodulated infrared signal.

A set of $\mathrm{C}$ programs was developed to control the instrument, and to record the data to disk. The simplest experiments consisted of sweeping the OPD while periodically sampling the demodulated output signal. Short $(\lesssim 100 \mu \mathrm{m})$ sweeps could be made using the PZT, while longer sweeps (up to $\sim 1 \mathrm{~cm}$ ) required moving the picomotor. Each scan measured the fringe envelope, providing both an estimate of the nuller performance (i.e., the null depth) and a qualitative estimate of the chromaticity of the fringe packet. Each picomotor step changed the OPD by a non-repeatable amount between $\sim 20-30 \mathrm{~nm}$, depending on the position of the drive screw. This stepsize was small enough to sample nulls deeper than $10^{-4}$, and the null depth was found to be repeatable from one picomotor scan to the next.

Stabilization of the null was done by slowly dithering the OPD using the PZT, i.e., moving it by a small fraction of a wavelength first to one side of the current estimated null position and then to the other. If the signal in the three positions decreased monotonically in one direction, then the estimate of the null position (and the center of the dither pattern) was adjusted in that direction by a distance equal to the dither amplitude. Sets of samples taken at the estimated null position were averaged, and their average and RMS scatter were saved. This crude stabilization algorithm proved to converge relatively slowly to a nearby null (the upper limit on its speed was set by the product of the dither amplitude and dither frequency), but it was also rather robust.

\section{EXPERIMENTAL RESULTS}

A variety of experiments were conducted with the tabletop nuller, starting with simple scans through the fringe envelope and later progressing to increasingly deep stabilized nulls. This section presents a selection of the more interesting data produced.

\subsection{Scans through Fringe Packets in Broadband and Narrowband Light}

Single scans were made stepping the OPD using the picomotor to move the fold mirror on one interferometer arm, and periodically sampling the demodulated signal from the lock-in amplifier. The scans were typically several hundred microns long, which was sufficient to cover the central fringe packet when a broadband source was used.

Here we present scans representing the extremes of the bandpasses we experimented with. The first scan was made using a thermal light source whose bandpass was limited by a $6.4 \%$ warm filter mounted in the collimated 


\section{Scan through Broadband Fringe}

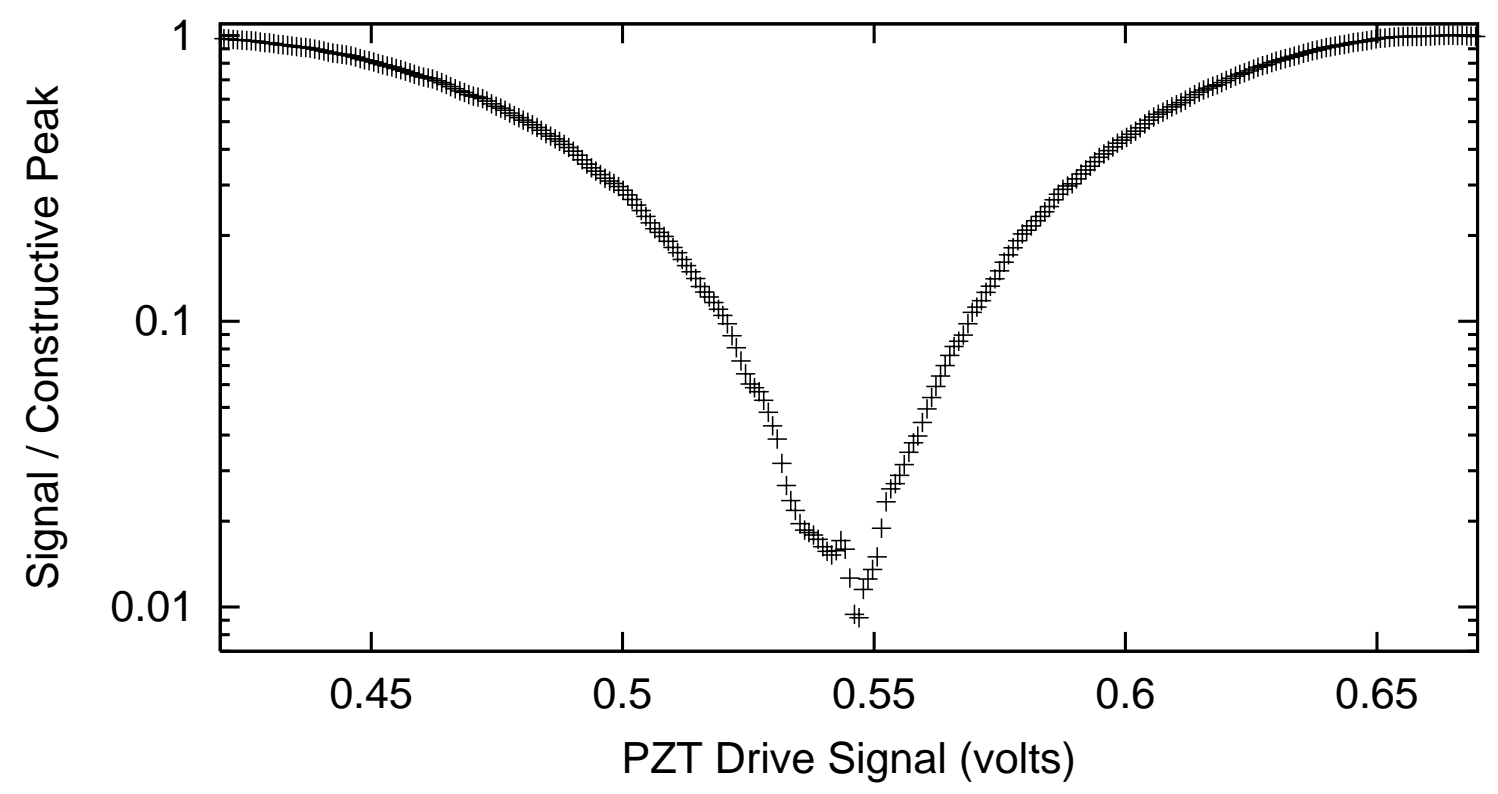

Figure 3. Scan through a thermal emitter fringe with the optical bandpass defined by a $6.4 \%$ interference filter. The signal at the bottom of the null is less than $10^{-2}$ times the signal at the constructive peak.

space upstream of the beamsplitter. It is shown in Figure 3. The horizontal axis is the control voltage sent to the PZT controller, and is representative of the OPD changes to the extent that the PZT is linear. The signal jumps slightly before, and again slightly after, the null position due to an automatic adjustment of the sensitivity of the lock-in amplifier. The best null depth is $10^{-2}$.

The second scan was made using the diode laser at a wavelength of $9.2 \mu \mathrm{m}$. It is plotted in Figure 4 with a logarithmic vertical scale to make the differences in the depths of the nulls visible. The narrow bandpass made the experiment relatively insensitive to residual chromatic dispersion, but there is still a noticeable asymmetry in the envelope. Despite this, the maximum fringe contrast is in excess of $10^{4}$.

\subsection{Null Stabilization Results}

Producing a stabilized null was an important goal for the tabletop infrared nuller experiment. As described in Section 4, it was accomplished in a simple way by comparing the demodulated signals as the OPD was dithered by a small fraction of a wavelength. Whenever the signal decreased monotonically in one direction during a dither pattern, the pattern was recentered on a position offset from the old one in that direction by one dither amplitude distance. The dither frequency was only a few Hertz, which was much less than the modulation frequency of the input light. Only the signal at the estimated position of the null was recorded. The stabilization system was used only with the laser, as the brightness of the thermal source was not adequate to allow deep nulls to be measured.

The result of one demonstration run of the stabilization system is plotted in Figure 5. The OPD was first tuned manually to a position close to a constructive fringe, and about 20 samples were recorded to establish a baseline. Then the OPD was manually tuned toward the position of the null, so that the demodulated signal intensity was reduced by a factor of about 10. At that point the system was left to seek the null. Once the null position was reached, the system held it stably for some 200 sample periods until the OPD was again manually tuned to the constructive fringe. The signal at the stabilized null position was not much larger than the detector noise, and as a result some of the individual measurements have the appearance of very deep nulls. Negative 


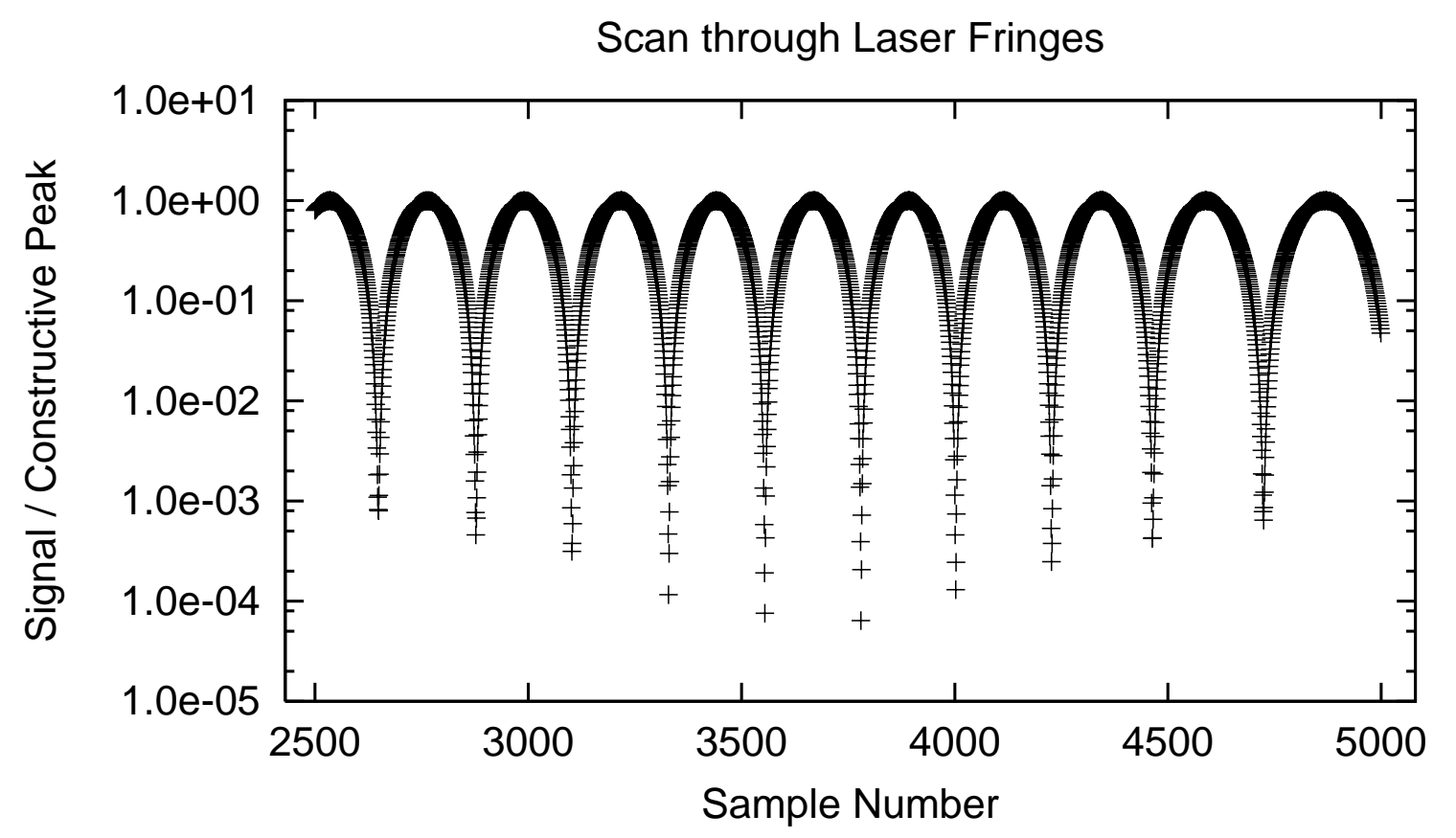

Figure 4. Scan through laser-light fringes with $9 \mu \mathrm{m}$ wavelength. Note the logarithmic vertical scale.

measurements resulting from noise fluctuations are not plotted because of the logarithmic vertical scale. Note that the null stabilization servo was active throughout the experiment, but was ineffectual when the OPD was not set close to a null.

A second experiment was run after some refinements were made to the optics. In particular, a polarizer in the collimated space between the laser and the beamsplitter was carefully adjusted to optimize the null depth (see Section 5.3 for details). The system was then manually tuned close to the null and allowed to seek the best OPD under servo control. Groups of samples at the null position were averaged to improve the effective sensitivity and to provide noise estimates. The results, plotted in Figure 6, show the deepest stabilized null achieved with the rooftop nuller, with the nulled signal averaging only $6 \times 10^{-5}$ of the constructive peak.

\subsection{Sensitivity to Polarization of the Input Beam}

The availability of the active stabilization system helped make it practical to explore the instrument's sensitivity to the polarization of the input light. The presence of dielectric coatings (including several antireflection coatings and beamsplitter passes seen by the light in each path from the input of the system to the output) and small misalignments of the rooftop mirrors would cause unequal relative delays in the wavefront phases of horizontally and vertically polarized light. This led us to suspect that deeper nulls could be produced using a light source which was purely vertically polarized rather than in a mixed state. This effect was thought to be potentially important even with the intrinsically polarized laser diode source, because the degree and direction of its polarization were not well known.

This effect was searched for by inserting a wire-grid linear polarizer (on a ZnSe substrate) in the collimated space upstream of the beamsplitter. The polarizer was made by Leonard Research Corp ${ }^{3}$. The polarizer mount permitted calibrated rotations. The absolute orientation was not precisely known, but the indicated zero was close to the polarization direction of the laser beam. The test consisted of locking the system on a null and monitoring the demodulated signal as the polarizer was set to a series of indicated position angles. This produced a smooth curve which was well fit by a quadratic close to its minimum (Figure 7), demonstrating both the polarization dependence and the effectiveness of the null stabilization servo. The relative alignment 


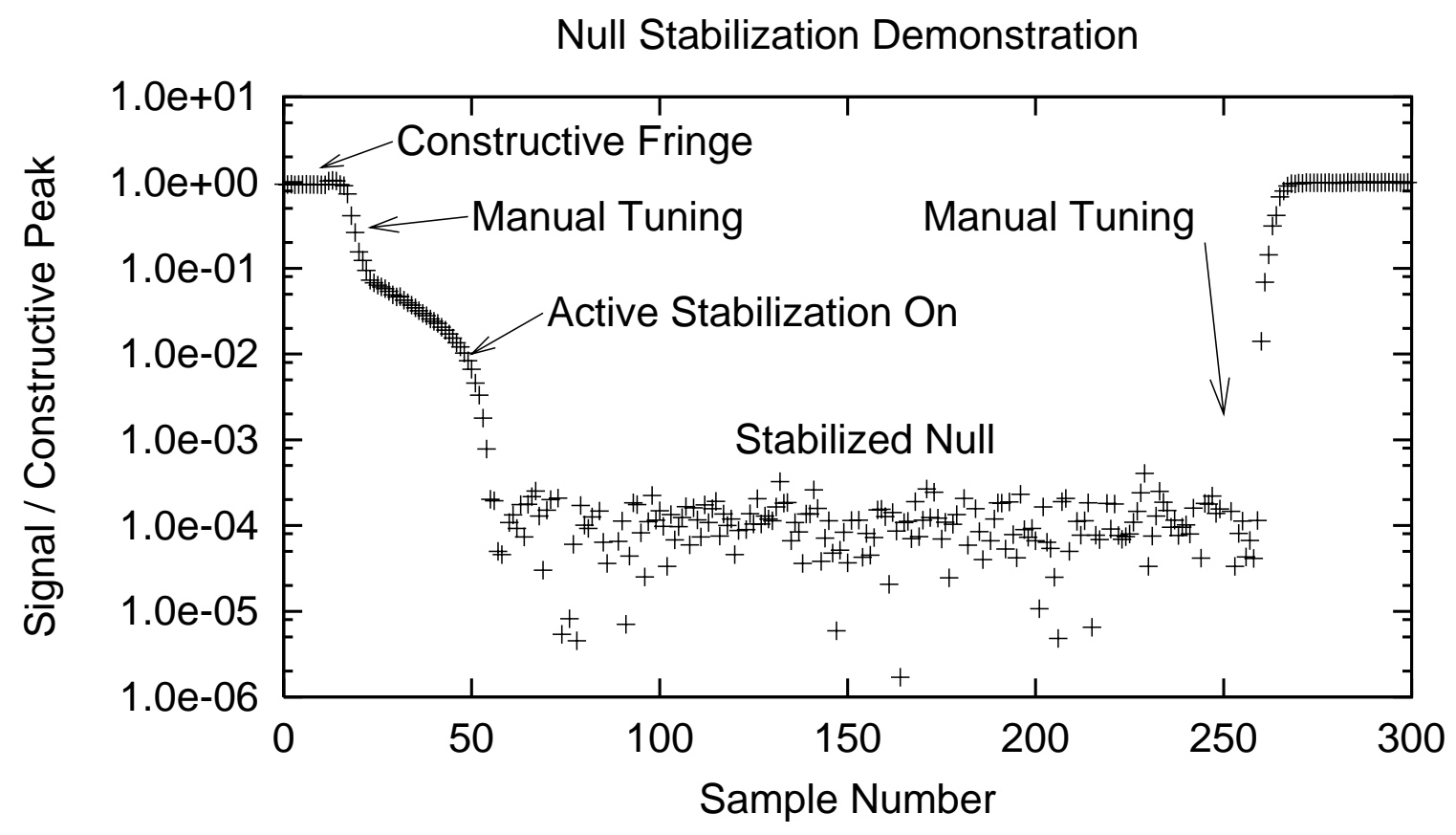

Figure 5. Demonstration of the stabilization of a laser-light null.

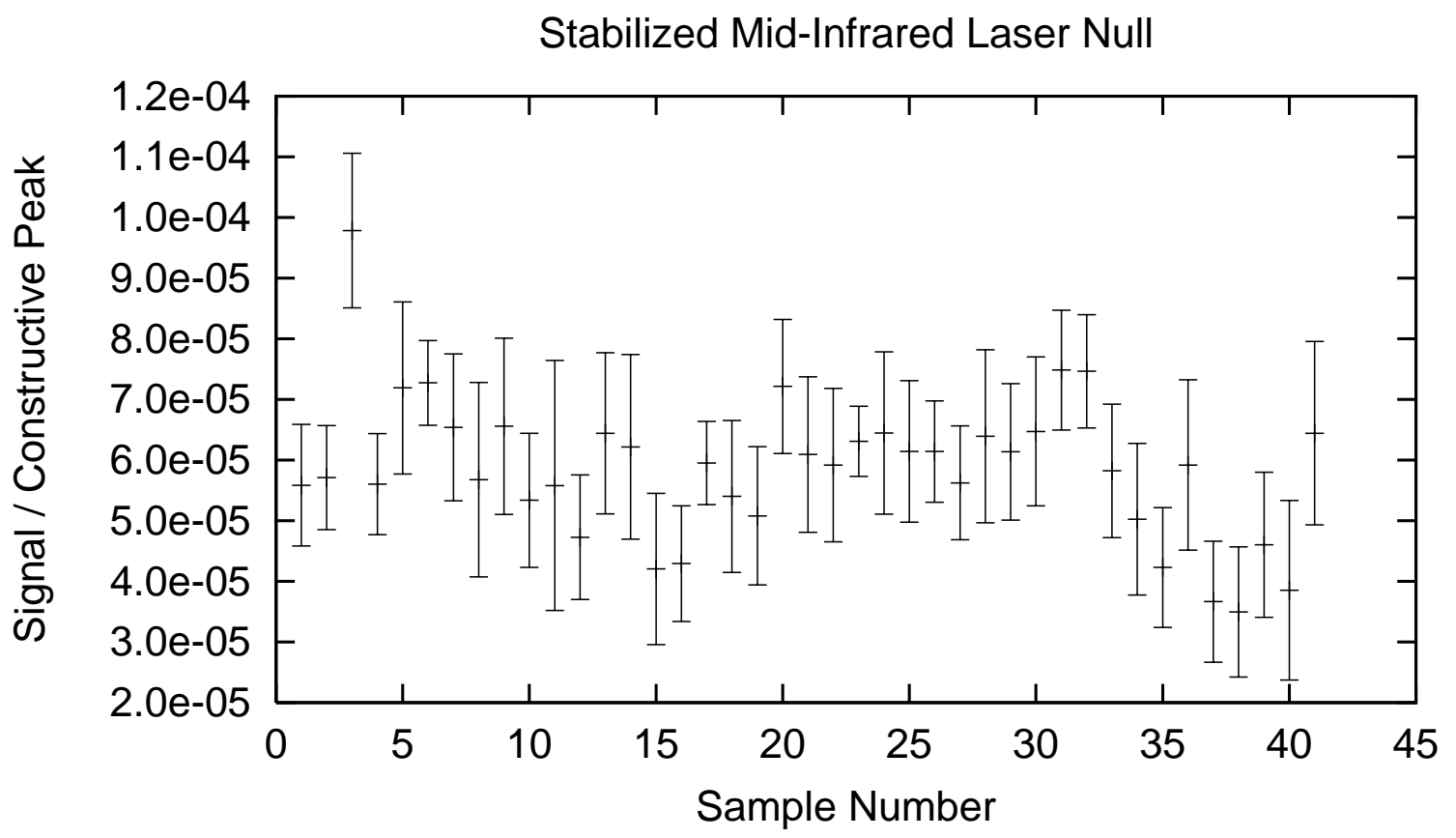

Figure 6. Stabilized null depth for a series of samples, using the laser diode source. 


\section{Null Depth vs Input Polarizer Angle}

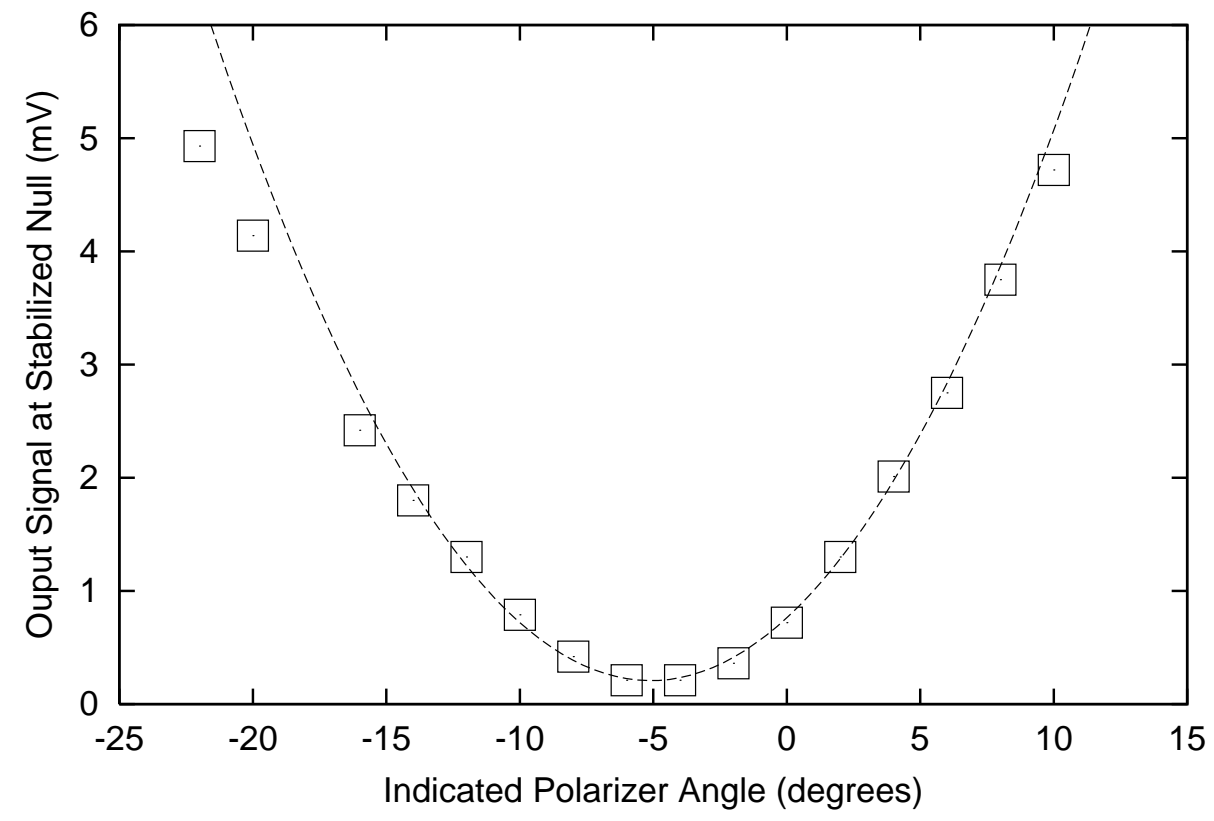

Figure 7. Signal measured at a stabilized null, as a function of polarizer position angle.

between the intrinsic polarization vector and the wire-grid polarizer presumably affected the throughput of the system at some level, but this effect should not be important close the minimum in the plotted curve.

\subsection{Balancing the Fluxes in the Arms}

An imbalance in the flux contributed by the two arms in the nuller leads to a leakage term which limits the depth of the null ${ }^{4}$. The flux in the each arm could be measured easily by blocking the other and observing the detected signal. When the system was well aligned, these were typically different from each other by about $10 \%$.

Two methods for equalizing the flux were investigated. The first was to deliberately misalign the arm showing the larger throughput. This method had been used successfully for the visible-light tabletop nulle ${ }^{2}$, but it never worked very well for the infrared nuller. We suspect that this is a result of the use of a pinhole rather than a single-mode fiber as a spatial filter, as the pinhole does not simply convert pointing offsets to througput losses, as a single-mode fiber does.

The second flux-balancing method was to partially block the beam in the arm with the higher flux by inserting an object into the collimated space. Both roughly circular irises and narrow rectangular singleelement "Venetian blinds" were used, with the latter giving more satisfactory results. This is probably due to the different ways the diffraction produced by these objects change the focussed image at the detector: the iris causes the Airy spot produced by a single arm to expand relative to that produced by the other, while the "Venetian blind," which consisted of a thin vertically-mounted strip of metal which could be rotated about its vertical axis to adjust its projected width, simply diffracted the excess light out of the beam while leaving the Airy spot essentially unchanged.

\section{CONCLUSIONS}

The experiments presented here have demonstrated that fringes with contrast in excess of $10^{4}$ can be produced and stabilized at mid-infrared wavelengths. The best fringes were made with vertically-polarized, narrow-band 
light from a diode laser source. Both chromatic and polarization effects were significant when a less ideal light source was used. Even so, broadband thermal nulls of $10^{-2}$ were seen.

To some extent the sensitivity to these adverse effects is inherent in the residual asymmetries present in the design of the rooftop beam combiner. The beam paths in the two arms must traverse several dielectric antireflection coatings, and in fact the number of such passages is larger by two in the arm containing the compensator plate. Further, the reflection at the beamsplitter in that arm occurs at an air/dielectric interface, while for the other arm it is at the interface between the beamsplitter and its substrate. It is therefore not surprising that the wavefronts contributed by the two arms have significantly different properties. This has led us to move on to a much more symmetrical beam-combiner design for further nulling work ${ }^{5}$.

\section{ACKNOWLEDGMENTS}

Funding was provided by NASA through the Keck Interferometer and Terrestrial Planet Finder projects. The research presented here was conducted at the Jet Propulsion Laboratory, California Institute of Technology, under contract with the National Aeronautics and Space Administration.

\section{REFERENCES}

1. The Terrestrial Planet Finder (TPF): a NASA Origins Program to Search for Habitable Planets. The TPF Science Working Group, JPL Publication 99-3, Edited by C.A. Beichman, N.J. Woolf and C.A. Lindensmith. May, 1999

2. E. Serabyn, J.K. Wallace, G.J. Hardy, E.G.H. Schmidlin, \& H. Nguyen, "Deep Nulling of Visible Laser Light", Applied Optics, 38, 7128-7132, 1999

3. M.J. Creech-Eakman, T. Leonard, J.M. Geis, J. M. \& E. Serabyn, "Comparison of commercially available polarizing gratings for mid-infrared studies", in Proc. SPIE "Interferometry in Optical Astronomy II" (this volume), 2002

4. E. Serabyn, "Nulling Interferometry: Symmetry Requirements and Experimental Results", in Proc. SPIE 4006, "Interferometry in Optical Astronomy," eds. P.J. Lena \& A. Quirrenbach, 328-339, 2000

5. E. Serabyn \& M.M. Colavita, "Fully Symmetric Nulling Beam Combiners ", Applied Optics, 40, 1668, 2001. 